\title{
Adaptation in the constancy of visual direction measured by a one-trial method'
}

\author{
HANS WALLACH AND KARL JOSEF FREY 2 \\ SWARTHMORE COLLEGE
}

\begin{abstract}
Adaptation to field displacement during head movements in the direction with the head rotation and in the direction against it was produced under otherwise identical conditions and compared; the field displacement rate was also varied. A rapid training procedure was used, and a novel one-trial test was employed that could measure the adaptation well enough to compare the effects of various training conditions. The one-trial test measured the magnitude of one of the manifestations of adaptation, the apparent displacement of a stationary target during head movements. This apparent horizontal target displacement was transformed into an oblique one by having the head movements that brought forth the apparent target displacement simultaneously cause an objective vertical target displacement. The slant of the resultant apparent motion path varied with the magnitude of the apparent horizontal target displacement. It was measured by having $S$ reproduce its slant angle. It was found that adaptation to field displacement in the direction with the head rotation was consistently greater than adaptation to the opposite displacement conditions. An explanation for this result is offered.
\end{abstract}

In previous work on the constancy of visual direction, ${ }^{3}$ a start was made in the investigation of adaptation to the regular displacements of the visual field during turning movements of the head such as occur when size lenses are worn. When such adaptation has taken place, it causes an objectively stationary visual field to appear to move during a head movement and this motion is in the direction opposite to the displacement to which $S$ had fully or partially adapted. Measurements of this adaptation were made by presenting a target visible in the dark that could be displaced dependent on S's head movements and by finding that ratio of target displacement to head rotation at which the target appeared to be stationary. Such measurements have been taken for adaptation to minification, which causes field displacement in the direction with the head movement (Wallach \& Kravitz, 1965a) and also for adaptation to magnification where the field displacement is in the direction against the head rotation (Wallach \& Kravitz, 1968). But these two opposite effects have never been produced under otherwise identical adaptation conditions and their measurements were thus not comparable. Such a comparison is interesting, however, for the following reason. Since the constancy of visual direction can be modified in as little as 5 min (Wallach \& Kravitz, 1965b), it seems reasonable to assume that the constancy which $S$ brings to the adaptation experiment, i.e., the fact that the visual field appears stationary during head movements, is also learned; that, in other words, experimental adaptation is a modification of the result of a previous adaptation to the natural conditions of everyday existence. What are these natural conditions that everybody adapts to?

In answering this question we have, for the moment, to give up the ordinary way of thinking about motion and rest. This thinking is based on the perception of a stationary environment where objects that are perceived to be in motion are, in fact, being displaced relative to that environment. This way of perceiving is in agreement with the practical physical facts that matter in our ordinary dealing with the objects in our environment. But it is, nevertheless, true that a stationary environment is a product of our perceptual processes. In visual stimulation, the stationary environment is represented as stationary only as long as the head is kept still. When the head is turned-because the eye sockets shift with the headstimulation conditions represent a stationary environment as being displaced relative to the head. Since, in the analysis of perceptual problems, conditions of stimulation rather than the physical environment are the primary facts, the displacement of the environment relative to eyes and head must be our starting point. With the head therefore taken as the frame of reference, a turning of the head to the right brings about a displacement of the visual field (relative to the head) to the left, and vice versa. Hence, the natural conditions to which everybody adapts consist in an environmental displacement in the direction against the head movement. Thus, the two kinds of experimental adaptation to artificially produced displacements of the optical, i.e., physical, environment differ in the way they relate to previous adaptation to the natural conditions; experimental adaptation to displacement against the head movement aims in the same direction as the previous adaptation, while the other kind, which occurs when minifiers are worn, develops in the opposite direction. A comparison between the two kinds of adaptation may, therefore, bear upon the relationship between experimental adaptation and previous adaptation whose result $\mathrm{S}$ brings to the experiment.

In the work to be reported, adaptation to displacement in the direction with the head movement and to displacement against the head movement was produced under conditions that were identical except with respect to direction. Also, the same group of Ss was employed in both forms of the experiment. This was done because all previous work showed very large individual differences in the amount of adaptation achieved under identical training conditions. The amount of displacement relative to the head movement, termed displacement ratio, to which Ss had to adapt was also varied; three different displacement ratios were used for each of the two directions, thus requiring each $S$ to participate in six different adaptation experiments. Since the effect of rapid training disappears more quickly and is therefore likely to affect subsequent adaptation training less, should an aftereffect of experimental adaptation on subsequent adaptation exist, we used the rapid training technique previously employed (Wallach \& Kravitz, 1965b).

The short-lived adaptation effects that result from rapid training present, however, a special problem: they are more difficult to measure in a uniform fashion, since they begin to deteriorate during the lengthy measuring procedures so far used by Wallach and Kravitz. A more rapid method of measuring adaptation was therefore developed for the present work; it consisted of a quantitative one-trial test. This test was based on the fact that an established adaptation effect causes a single stationary target to appear to move during a head turning in the direction opposite to the relative displacement to which $S$ had adapted. The extent of this apparent displacement of a stationary target varies with extent of the head rotation and with the amount of adaptation attained. The latter follows from the manner in which adaptation was previously measured: the range of displacement ratios that 
lead to apparent immobility of the objectively moving target was determined (no-motion range) and its midpoint computed. The latter, the no-motion point, was the measure of adaptation. The larger the displacement ratio yielding this no-motion point, that is, the farther in the scale of displacement ratios (DR) the apparent no-motion point fell from the objective no-motion point (zero DR), the greater the adaptation measured. It is most likely that the larger the objective target displacement relative to the head movement that leads to apparent target immobility, the larger should be the apparent displacement of an objectively stationary target. And, since the objective target displacement resulting in apparent target rest is the larger the greater the adaptation, the apparent displacement of a stationary target should also increase with the amount of adaptation achieved. Furthermore, since the objective target displacement that leads to perceived target rest is relative to the amount of head movement, and is measured as a displacement ratio, it might be expected that the apparent displacement of a stationary target that occurs during head movements is likewise proportionate to the head movement; hence, our previous statement that the extent of the apparent displacement varies also with the extent of the head movement. Thus, if we want to use this apparent displacement of a stationary target as a measure of adaptation, we must either keep the extent of head movement constant or contrive to elicit an estimate that represents the ratio of the apparent displacement to the extent of the head movement.

We took the latter course. Instead of presenting $S$ with a stationary target, the test was so arranged that S's head movements caused the target spot to become vertically displaced; when $S$ turned his head to the right the target moved objectively upward, and left turning made the target move down. Thus, the same head movement that brought forth the apparent horizontal target displacement caused by the adaptation effect would simultaneously elicit an objective vertical displacement for the target. We had hoped, and indeed found, that the two displacements, although one was objective and the other apparent, add vectorially to produce an apparent oblique target motion: after the adaptation training Ss reported target motion at a slant, and they were able to reproduce the slant of the apparent motion path by setting a rod that could be turned in the frontal plane.

This slant estimate represents the extent of the apparent horizontal target displacement, because the tangent of the slant angle should equal the ratio of the extent of the apparent horizontal displacement over the extent of the objective vertical displacement. The apparatus used in this test was such that the angle of the extent of the vertical displacement was a constant fraction of the extent of the head rotation; in the present work it was always $40 \%$ of the latter. Since the same head movement that caused the apparent horizontal target displacement governed the objective vertical target displacement, the slant of the apparent path of the target motion represents the extent of the apparent horizontal target displacement also as a fraction of the head rotation, that is, as a displacement ratio.

Before the adaptation experiment proper, we explored S's ability to detect deviation from verticality of the target's motion path and to make estimates of the slants of a number of different motion paths. Our apparatus was so modified that the target could objectively move at a certain slant when $S$ turned his head. The apparatus would simultaneously displace the target vertically at a constant ratio of $40 \%$ of the angle of the head rotation and horizontally at a rate that could be varied from trial to trial. The resultant of the two simultaneous displacements was a slanted motion path whose slant angle depended on the rate of the horizontal displacement. When these trials had shown that variations in the displacement ratio in the horizontal motion component caused corresponding changes in the slant estimates, we proceeded to use this technique in tests of adaptation, where the horizontal component of the slanted motion path was supplied by the adaptation effect.

\section{APPARATUS}

The measuring device previously used by Wallach and Kravitz (1968) was modified to produce vertical as well as horizontal target displacement. The projector beam that cast the target disk on the screen in front of $S$ was successively reflected by two mirrors, a larger one that turned about a horizontal shaft and the one previously used that turned about a vertical axis and was mounted on the output shaft of the variable transmission. While the latter's angular displacement relative to the rotation of the input shaft was variable and controlled by setting the transmission ratio to a desired value, the larger mirror turned in fixed proportion to the shaft, causing a vertical target displacement of $40 \%$ of the head rotation. Either one of the mirror shafts could be disconnected from the turning parts of the apparatus and fixed in a constant position.

The $1 / 4$-in. metal rod used by $S$ to indicate the slant of the target motion was 22.5 in. long. Attached perpendicularly at its midpoint was a short shaft that served as rotation axis for the rod. It had its bearing in the center of a white board 28 in. high and $22 \mathrm{in.}$ wide. A long pointer was fixed on the other end of the shaft in the board's rear, permitting accurate reading of the rod position from a large scale. The board was mounted on a stand so high that the rod's shaft was at the level of S's eyes and was placed near the testing apparatus so that, by turning his head to the side, $S$ could face the board and move the rod by hand.

\section{PRELIMINARY EXPERIMENTS}

(1) To test our Ss' ability to detect slanted target motion we followed the procedure used by Wallach and Kravitz in measuring the uncertainty range in the constancy of visual direction. Such measurements were concerned with the detection of objective, horizontal target displacements during head movements. In the present experiment an unchanging vertical displacement amounting to $40 \%$ of the head rotation angle was added to the variable horizontal displacement. As the displacement ratio of the horizontal target displacement was varied from displacement with the head movement through target immobility to displacement against the head movement, the resultant motion path of the target varied from slant to the right through verticality to slant to the left. Instead of reporting whether the target seemed to move or to be stationary as was done in the previous measurements, $S$ was asked whether its motion path appeared vertical or slanted, in other words, judgments of verticality vs slant replaced judgments of target rest or motion; in all other respects the procedure remained the same. The mean uncertainty range for perceived verticality of target motion was found to be $8.6 \%$ DR (14 Ss). This was $2 \%$ DR larger than the mean uncertainty range of $6.6 \%$ DR for apparent target rest obtained by Wallach and Kravitz (1965a) with naive Ss, and much larger than the mean of $3.86 \%$ DR obtained by the same Es with 14 practiced Ss (1968).

(2) We determined our Ss' ability to give estimates of the slant of the motion path by presenting them with eight different motion paths, of four different slant angles in each of the two slant directions, and had them reproduce the apparent slant of each motion path with the tilting rod. The different slant angles were produced by setting the horizontal target displacement to $5.3,10.6,15.9$, and $21.2 \% \mathrm{DR}$. With the simultaneous vertical displacement a constant $40 \% \mathrm{DR}$, the resultant motion paths had slant angles of $7.5 \mathrm{deg}, 14.8 \mathrm{deg}$, 
Table 1

Mean Slant Estimates for Eight Motion Paths

\begin{tabular}{lllllllll}
$\begin{array}{l}\text { Slant Direction } \\
\text { Horizontal displacement }\end{array}$ & \multicolumn{4}{c}{$\begin{array}{c}\text { Counter Clockwise } \\
\text { Against }\end{array}$} & \multicolumn{3}{c}{. } & \multicolumn{3}{c}{$\begin{array}{c}\text { Clockwise } \\
\text { With }\end{array}$} \\
\hline Displacement rate & $21.2 \%$ & $15.9 \%$ & $10.6 \%$ & $5.3 \%$ & $5.3 \%$ & $10.6 \%$ & $15.9 \%$ & $21.2 \%$ \\
Objective slant angle & $27.9 \mathrm{deg}$ & $21.7 \mathrm{deg}$ & $14.8 \mathrm{deg}$ & $7.5 \mathrm{deg}$ & $7.5 \mathrm{deg}$ & $14.8 \mathrm{deg}$ & $21.7 \mathrm{deg}$ & $27.9 \mathrm{deg}$ \\
Slant estimates & $20.5 \mathrm{dge}$ & $15.4 \mathrm{deg}$ & $9.6 \mathrm{deg}$ & $3.9 \mathrm{deg}$ & $3.7 \mathrm{deg}$ & $10.0 \mathrm{deg}$ & $15.0 \mathrm{deg}$ & $19.8 \mathrm{deg}$ \\
\hline
\end{tabular}

$21.7 \mathrm{deg}$, and $27.9 \mathrm{deg}$. The slant direction was controlled by causing the horizontal target displacement to be in the direction with or against the head movement.

Sitting under the measuring device, his head attached to its input shaft, $\mathrm{S}$ first turned his head to face the rod and adjusted it to the apparent vertical position. E read the setting and if it deviated from the true vertical, which happened only rarely, took down the rod position. Then, with the room darkened and the horizontal target displacement set to one of the predetermined values, $\mathrm{S}$ made three full head movements, thereby causing three up-and-down excursions of the target spot. He turned his head toward the rod, and, with the light turned on, made a rod setting. His slant estimate was the difference in degrees between his vertical setting and his slant setting. The sequence of presentation of the eight different motion paths was varied from $S$ to $S$, but so that a slant in one direction was always immediately followed by a slant in the other direction. Each motion path was presented twice in the sequence of presentation and the average of the two estimates became S's score for that particular slant angle. In anticipation of a procedure followed in the main experiment, the starting position of the rod when $\mathrm{S}$ made his setting was always vertical.

The results are presented in Table 1. A comparison of the means of the slant estimates with the slant angles of the given target paths shows an underestimation of the slant angle. While this underestimation increased with the given slant angles, it was by no means proportional to them. Whereas the slant estimates given for particular motion paths by different Ss showed rather large variations, estimates by individual Ss showed good discrimination of the eight objective slants. For a total of 84 pairs of slant estimates given by individual Ss for adjacent objective slants, only two errors occurred such that the greater of the two objective slants was judged the smaller and vice versa.

\section{PROCEDURE IN THE MAIN EXPERIMENTS}

Rapid adaptation to field displacement with and against the head movement was compared for three rates of field displacement: 20,40 , and $80 \%$ DR. Thus, each of our $14 \mathrm{Ss}$, the same who had been used in the second preliminary experiment and therefore mastered the rod setting technique, participated in six adaptation experiments that differed in direction or rate of the field displacement.

Each such experiment began with an initial rod setting. The mirror that caused the horizontal target displacement was arrested and the vertically displacing mirror was connected to the input shaft so that head turning produced objectively vertical target displacement. $S$ had to make one rod setting that reproduced the apparent direction of this motion path. S was allowed two full head movements before setting the rod to the perceived target motion direction, and this procedure was followed throughout the experiment. The adaptation period, which now followed, was similar to the one used by Wallach and Kravitz (1965b) for rapid adaptation in that S simply remained in the test apparatus, which was set for a high displacement ratio, continuously turned his head back and forth and observed the moving target spot. It differed in that training lasted for exactly 100 full head movements and that the various displacement ratios for which the transmission was set were much smaller than the one ratio previously used. To change the apparatus to the adaptation conditions the vertically displacing mirror simply was disengaged and the variable transmission was set for one of the six displacement ratios stated above. After the room was darkened, $\mathrm{S}$ was asked to turn his head back and forth at a comfortable rate and pay attention to the moving target spot. When this adaptation period, which lasted on the average $3 \mathrm{~min}$, ended, the apparatus was again arranged for objectively vertical target motion, with $\mathrm{S}$ sitting still and with eyes closed. Directly after this change-over, which took very little time, $S$ made one slant setting. The difference in slant angle between this setting and the preadaptation setting is the measured adaptation effect.

Casual observations by Wallach and Kravitz had shown that after termination of the training period the adaptation effect gradually diminishes and disappears. The question arises whether encounters with normal field displacements during head movements are responsible for this or whether the mere lapse of time serves to diminish experimental adaptation and to reestablish the normal constancy of visual direction. Our one-trial measuring method is particularly suitable to answering this question, for it is uniform and takes little time. Therefore, following the postadaptation rod setting, S was asked to close his eyes and to sit still, with his head in normal position. After 5 min another rod setting was made.

Immediately after this test for the decline of the adaptation effect with lapse of time, $\mathrm{S}$ was asked to turn his head back and forth five times while observing the target spot that was now objectively entirely immobile, and this was followed directly by another rod setting. This procedure gave us a rough idea of the effectiveness of a brief exposure to conditions that would tend to reestablish the normal constancy of visual direction.

An individual adaptation experiment ended here. $S$ was asked to get up and move about freely for $20 \mathrm{~min}$. After this rest period another one of the six adaptation experiments began. It was always the one with the same rate of field displacement that was used in the first adaptation of the day's session and in the opposite direction. Only two experiments were run on the same day; there were thus three experimental sessions in all; they were separated by at least 3 days. Except for the rule just stated, the sequence of adaptation was varied from $S$ to $S$ and satisfactorily balanced. When the initial rod setting of the second adaptation experiment of a given session raised doubt about whether all the effect of the preceding adaptation had disappeared, the experiment was broken off and was started at another time.

\section{RESULTS}

The mean adaptation effects obtained under the six training conditions are listed in the first row of Table 2 . In the case of both displacement directions, the effect increased as the displacement rate of the target rose from $20 \%$ to $40 \%$ of the head rotation, and this increase is significant at the .05 level. A further rise in the displacement ratio, to $80 \%$, did not produce a comparable increase in the adaptation effect. Adaptation to target displacement in the direction with the head movement was consistently higher than adaptation to displacement against the head turning, a difference significant at better than the .005 level. $^{4}$ 
Table 2

Mean Effects of Six Conditions of Adaptation in Degrees of Slant and Decline of the Effects after 5 min Rest and after Five Additional Head Movements (HM)

\begin{tabular}{lcccccc}
\hline $\begin{array}{l}\text { Adaptation direction } \\
\text { Displacement rate }\end{array}$ & $20 \%$ & $40 \%$ & $80 \%$ & $20 \%$ & $40 \%$ & $80 \%$ \\
\hline $\begin{array}{l}\text { Adaptation effect } \\
\text { Slant of rod setting }\end{array}$ & 6.82 & 8.65 & 9.42 & 4.28 & 6.68 & 6.29 \\
$\quad$ & & & & & & \multicolumn{3}{c}{ With } \\
$\quad$ after adaptation & 6.71 & 8.61 & 9.46 & 4.21 & 6.68 & 6.04 \\
$\quad$ min later & 3.86 & 5.43 & 5.18 & 1.18 & 3.39 & 2.43 \\
Proportionate decline & $42.5 \%$ & $36.9 \%$ & $45.2 \%$ & $72 \%$ & $49.2 \%$ & $59.7 \%$ \\
$\quad \begin{array}{l}\text { Slant of rod setting } \\
\quad \text { after 5 HM }\end{array}$ & 2.54 & 3.79 & 2.96 & .79 & 2.07 & 1.50 \\
\hline
\end{tabular}

There was a large and highly significant decline in the adaptation effect after the 5-min period of rest, as shown by a comparison of the means of the slant settings made immediately after adaptation and of those made 5 min later (second and third row in Table 2). In the fourth row of Table 2 this decline is given as a percentage of the effect measured immediately after the adaptation period. Presented in this fashion the decline is consistently greater in the case of adaptations to displacements in the direction against the head movement, and this difference is significant at the .01 level. The subsequent five head movements caused a sizeable further decline of what was left of the experimental adaptation (fifth row of Table 2). This brief exposure to conditions tending to reestablish the normal constancy of visual direction was quite effective $(p<.001)$. It should be mentioned that our slant tests also represented the conditions for the reestablishment of the normal constancy, because here, too, the target spot was stationary where the left-right dimension was concerned while $\mathrm{S}$ turned his head twice. (The vertical target displacement that occurred in these tests is not relevant.) Therefore, part of the decrease in the experimental adaptation measured after the 5 -min rest period must be ascribed to the additional test.

\section{DISCUSSION}

The many significant differences that were obtained in these experiments are a tribute to the one-trial test used here for the first time. This test permits very brief and therefore more uniform training periods, which cause adaptation effects that decline rapidly and disappear completely, making feasible the use of the same Ss for all the experimental variations.

That experimental adaptation to target displacement with the head movement is consistently and very significantly greater than adaptation that goes in the opposite direction is the main result of our experiment. As explained above, the two kinds of experimental adaptation, which were here produced under corresponding conditions, differ in the manner in which they relate to the adaptation that Ss bring to our experiment. The latter, the adaptation to the normal field displacements relative to the head caused by head movements under natural conditions, is an adaptation to displacements in the direction against the head movement. Experimental adaptation to target displacement against the head movement is thus a development that goes in the same direction as adaptation to natural conditions and thus enhances it, whereas experimental adaptation to objective displacement with the head movement goes in the direction opposite to natural adaptation and diminishes it. That the greater experimental adaptation was obtained under the latter condition makes sense. It seems reasonable that a change that diminishes a previously established adaptation effect develops more rapidly than the opposite one that requires a further enhancement of the previously established adaptation. That experimental adaptation to objective displacement against the head movement declines more rapidly than the opposite adaptation is a finding that should probably be considered in the same context.

\section{REFERENCES}

WALLACH, H., \& KRAVITZ, J. H. The measurement of the constancy of visual direction and of its adaptation. Psychonomic Science, 1965a, 2, 217-218.

WALLACH, H., \& KRAVITZ, J. H. Rapid adaptation in the constancy of visual direction with active and passive rotation. Psychonomic Science, $1965 \mathrm{~b}, 3,165-166$.

WALLACH, H., \& KRAVITZ, J. H. Adaptation in the constancy of visual direction tested by measuring the constancy of auditory direction. Perception \& Psychophysics, 1968, 4, 299-303.

\section{NOTES}

1. The work was supported by Grant 11089 by the National Institute of Mental Health.

2. Address: Department of Psychology, Swarthmore College, Swarthmore, Pennsylvania 19081

3. A lengthy explication of this concept, of the device used for measuring the constancy of visual direction, and of the effect of size lenses in causing displacements of the visual field is given in Wallach and Kravitz (1968).

4. It may be worth mentioning that there exists a correlation indicating that Ss who showed a strong adaptation effect to displacement in the direction with the head movement also tended to show a strong effect when displacement was in the direction against the head movement; $\mathrm{r}=.54, \mathrm{p}<.05$

(Accepted for publication December 24, 1968.) 